\title{
Ni-Serrin type equations arising from capillarity phenomena with non-standard growth
}

\section{Mustafa Avci*}

\section{"Correspondence:}

mustafa.avci@batman.edu.tr Department of Management, Faculty of Economics and Administrative Sciences, Batman University, Batman, Turkey

\begin{abstract}
In the present paper, in view of the variational approach, we discuss a Ni-Serrin type equation involving non-standard growth condition and arising from the capillarity phenomena. Establishing some suitable conditions, we prove the existence and multiplicity of solutions.
\end{abstract}

MSC: 35D05; 35J60; 35J70

Keywords: $p(x)$-Laplacian; variable exponent Sobolev space; mountain pass theorem; genus theory; variational method; capillarity phenomena

\section{Introduction}

We study the existence and multiplicity of solutions for a Ni-Serrin type equation involving non-standard growth condition and arising from capillarity phenomena of the following type:

$$
\left\{\begin{array}{l}
-M(L(u)) \operatorname{div}\left(|\nabla u|^{p(x)-2} \nabla u+\frac{|\nabla u|^{2 p(x)-2} \nabla u}{\sqrt{1+|\nabla u|^{2 p(x)}}}\right)=f(x, u), \quad x \in \Omega, \\
u=0, \quad x \in \partial \Omega
\end{array}\right.
$$

where $\Omega \subset \mathbb{R}^{N}$ is a bounded domain with smooth boundary $\partial \Omega, p \in C(\bar{\Omega})$ such that $1<$ $p(x)<N$ for any $x \in \bar{\Omega}$ and $L(u):=\int_{\Omega} \frac{|\nabla u|^{p(x)}+\sqrt{1+|\nabla u|^{2 p(x)}}}{p(x)} d x$.

Capillarity can be briefly explained by considering the effects of two opposing forces: adhesion, i.e., the attractive (or repulsive) force between the molecules of the liquid and those of the container; and cohesion, i.e., the attractive force between the molecules of the liquid. The study of capillary phenomena has gained some attention recently. This increasing interest is motivated not only by fascination in naturally-occurring phenomena such as motion of drops, bubbles and waves but also its importance in applied fields ranging from industrial and biomedical and pharmaceutical to microfluidic systems.

The study of ground states for equations of the form

$$
-\operatorname{div}\left(\frac{\nabla u}{\sqrt{1+|\nabla u|^{2}}}\right)=f(u) \text { in } \mathbb{R}^{N}
$$

where $G(u)=\frac{\nabla u}{\sqrt{1+|\nabla u|^{2}}}$ is the Kirchhoff stress term and the source term $f$ was very general, was initiated by $\mathrm{Ni}$ and Serrin [1,2]. Moreover, radial solutions of the problem (1.1) have

\section{空 Springer}

(c) 2013 Avci; licensee Springer. This is an Open Access article distributed under the terms of the Creative Commons Attribution License (http://creativecommons.org/licenses/by/2.0), which permits unrestricted use, distribution, and reproduction in any medium, provided the original work is properly cited. 
been studied in the context of the analysis of capillarity surfaces for a function of the form $f(u)=k u, k>0$ (see [3-5]). Recently, in [6] Rodrigues studied a version of the problem (P) for the case $M(L(u)) \equiv 1$ and $f(x, u) \equiv \lambda f(x, u), \lambda>0$.

We note that if we choose the functional $L(u)$ as $\int_{\Omega} \frac{\mid \nabla u p^{p(x)}}{p(x)} d x$ in $(\mathbf{P})$, then we get the problem

$$
\left\{\begin{array}{l}
-M\left(\int_{\Omega} \frac{|\nabla u|^{p(x)}}{p(x)} d x\right) \operatorname{div}\left(|\nabla u|^{p(x)-2} \nabla u\right)=f(x, u) \text { in } \Omega \\
u=0 \text { on } \partial \Omega
\end{array}\right.
$$

which is called the $p(x)$-Kirchhoff type equation [7-9]. In this case, the problem (1.2) indicates a generalization of a model, the so-called Kirchhoff equation, introduced by Kirchhoff in [10]. To be more precise, Kirchhoff established a model given by the equation

$$
\rho \frac{\partial^{2} u}{\partial t^{2}}-\left(\frac{P_{0}}{h}+\frac{E}{2 l} \int_{0}^{l}\left|\frac{\partial u}{\partial x}\right|^{2} d x\right) \frac{\partial^{2} u}{\partial x^{2}}=0
$$

where $\rho, P_{0}, h, E, l$ are constants, which extends the classical D'Alambert wave equation by considering the effects of the changes in the length of the strings during the vibrations. A distinguishing feature of Kirchhoff equation (1.3) is that the equation contains a nonlocal coefficient $\frac{P_{0}}{h}+\frac{E}{2 l} \int_{0}^{l}\left|\frac{\partial u}{\partial x}\right|^{2} d x$ which depends on the average $\frac{E}{2 l} \int_{0}^{l}\left|\frac{\partial u}{\partial x}\right|^{2} d x$ of the kinetic energy $\frac{1}{2}\left|\frac{\partial u}{\partial x}\right|^{2}$ on $[0, l]$, and hence the equation is no longer a pointwise identity.

The nonlinear problems involving the $p(x)$-Laplacian operator, that is, $\operatorname{div}\left(|\nabla u|^{p(x)-2} \nabla u\right)$, are extremely attractive because they can be used to model dynamical phenomena which arise from the study of electrorheological fluids or elastic mechanics, in the modeling of stationary thermo-rheological viscous flows of non-Newtonian fluids and in the mathematical description of the processes filtration of an ideal barotropic gas through a porous medium [11-15]. The detailed application backgrounds of the $p(x)$-Laplacian can be found in $[16-20]$ and references therein.

\section{Abstract framework and preliminary results}

We state some basic properties of the variable exponent Lebesgue-Sobolev spaces $L^{p(x)}(\Omega)$ and $W^{1, p(x)}(\Omega)$, where $\Omega \subset \mathbb{R}^{N}$ is a bounded domain (for details, see [21-24]).

Set

$$
C_{+}(\bar{\Omega})=\{p ; p \in C(\bar{\Omega}), \inf p(x)>1 \text { for all } x \in \bar{\Omega}\}
$$

Let $p \in C_{+}(\bar{\Omega})$ and denote

$$
p^{-}:=\inf _{x \in \bar{\Omega}} p(x) \quad \text { and } \quad p^{+}:=\sup _{x \in \bar{\Omega}} p(x)
$$

For any $p \in C_{+}(\bar{\Omega})$, we define the variable exponent Lebesgue space by

$$
L^{p(x)}(\Omega)=\left\{u \mid u: \Omega \rightarrow \mathbb{R} \text { is measurable, } \int_{\Omega}|u(x)|^{p(x)} d x<\infty\right\}
$$


then $L^{p(x)}(\Omega)$ endowed with the norm

$$
|u|_{p(x)}=\inf \left\{\mu>0: \int_{\Omega}\left|\frac{u(x)}{\mu}\right|^{p(x)} d x \leq 1\right\}
$$

becomes a Banach space.

Proposition $1[22,24]$ For any $u \in L^{p(x)}(\Omega)$ and $v \in L^{p^{\prime}(x)}(\Omega)$, we have

$$
\left|\int_{\Omega} u v d x\right| \leq\left(\frac{1}{p^{-}}+\frac{1}{\left(p^{-}\right)^{\prime}}\right)|u|_{p(x)}|v|_{p^{\prime}(x)}
$$

where $L^{p^{\prime}(x)}(\Omega)$ is a conjugate space of $L^{p(x)}(\Omega)$ such that $\frac{1}{p(x)}+\frac{1}{p^{\prime}(x)}=1$.

The modular of $L^{p(x)}(\Omega)$, which is the mapping $\rho: L^{p(x)}(\Omega) \rightarrow \mathbb{R}$, is defined by

$$
\rho(u)=\int_{\Omega}|u(x)|^{p(x)} d x
$$

for all $u \in L^{p(x)}(\Omega)$.

Proposition $2[22,24]$ If $u, u_{n} \in L^{p(x)}(\Omega)(n=1,2, \ldots)$, then the following statements are equivalent:

(i) $\lim _{n \rightarrow \infty}\left|u_{n}-u\right|_{p(x)}=0$;

(ii) $\lim _{n \rightarrow \infty} \rho\left(u_{n}-u\right)=0$;

(iii) $u_{n} \rightarrow u$ in measure in $\Omega$ and $\lim _{n \rightarrow \infty} \rho\left(u_{n}\right)=\rho(u)$.

Proposition $3[22,24]$ If $u, u_{n} \in L^{p(x)}(\Omega)(n=1,2, \ldots)$, we have

(i) $|u|_{p(x)}<1(=1$; $>1) \Leftrightarrow \rho(u)<1(=1$; $>1)$;

(ii) $|u|_{p(x)}>1 \Longrightarrow|u|_{p(x)}^{p^{-}} \leq \rho(u) \leq|u|_{p(x)}^{p^{+}} ;|u|_{p(x)}<1 \Longrightarrow|u|_{p(x)}^{p^{+}} \leq \rho(u) \leq|u|_{p(x)}^{p^{-}}$;

(iii) $\lim _{n \rightarrow \infty}\left|u_{n}\right|_{p(x)}=0 \Leftrightarrow \lim _{n \rightarrow \infty} \rho\left(u_{n}\right)=0$;

$$
\lim _{n \rightarrow \infty}\left|u_{n}\right|_{p(x)}=\infty \Leftrightarrow \lim _{n \rightarrow \infty} \rho\left(u_{n}\right)=\infty .
$$

The variable exponent Sobolev space $W^{1, p(x)}(\Omega)$ is defined by

$$
W^{1, p(x)}(\Omega)=\left\{u \in L^{p(x)}(\Omega):|\nabla u| \in L^{p(x)}(\Omega)\right\}
$$

with the norm

$$
\|u\|_{1, p(x)}=|u|_{p(x)}+|\nabla u|_{p(x)}
$$

for all $u \in W^{1, p(x)}(\Omega)$.

The space $W_{0}^{1, p(x)}(\Omega)$ is defined as the closure of $C_{0}^{\infty}(\Omega)$ in $W^{1, p(x)}(\Omega)$ with respect to the norm $\|u\|_{1, p(x)}$. For $u \in W_{0}^{1, p(x)}(\Omega)$, we can define an equivalent norm

$$
\|u\|=|\nabla u|_{p(x)}
$$


since the Poincaré inequality holds, i.e., there exists a positive constant $C_{1}>0$ such that

$$
|u|_{p(x)} \leq C_{1}|\nabla u|_{p(x)}
$$

for all $u \in W_{0}^{1, p(x)}(\Omega)[18,24]$.

Proposition $4[22,24]$ If $1<p^{-} \leq p^{+}<\infty$, then the spaces $L^{p(x)}(\Omega), W^{1, p(x)}(\Omega)$ and $W_{0}^{1, p(x)}(\Omega)$ are separable and reflexive Banach spaces.

Proposition 5 [22, 24] Let $q \in C_{+}(\bar{\Omega})$. If $q(x)<p^{*}(x)$ for all $x \in \bar{\Omega}$, then the embedding $W^{1, p(x)}(\Omega) \hookrightarrow L^{q(x)}(\Omega)$ is compact and continuous, where $p^{*}(x)=\frac{N p(x)}{N-p(x)}$ if $p(x)<N$ and $p^{*}(x)=+\infty$ if $p(x) \geq N$.

Proposition 6 [18] Let X be a Banach space and let define the functional $\Lambda=\int_{\Omega} \frac{|\nabla u|^{p(x)}}{p(x)} d x$. Then $\Lambda: X \rightarrow \mathbb{R}$ is convex. The mapping $\Lambda^{\prime}: X \rightarrow X^{*}$ is a strictly monotone, bounded homeomorphism of $\left(S_{+}\right)$type, namely

$$
u_{n} \rightarrow u \quad \text { in } X \quad \text { and } \quad \varlimsup_{n \rightarrow \infty}\left\langle\Lambda^{\prime}\left(u_{n}\right), u_{n}-u\right\rangle \leq 0 \quad \text { implies } \quad u_{n} \rightarrow u \quad \text { in } X \text {. }
$$

Definition 7 Let $X$ be a Banach space and $J: X \rightarrow \mathbb{R}$ be a $C^{1}$-functional. We say that a functional $J$ satisfies the Palais-Smale condition ((PS) for short) if any sequence $\left\{u_{n}\right\}$ in $X$, such that $\left\{\left(u_{n}\right)\right\}$ is bounded and $J^{\prime}\left(u_{n}\right) \rightarrow 0$ as $n \rightarrow \infty$, admits a convergent subsequence.

We say that $u \in W_{0}^{1, p(x)}(\Omega)$ is a weak solution of $(\mathbf{P})$ if

$$
M(L(u)) \int_{\Omega}\left(|\nabla u|^{p(x)-2} \nabla u+\frac{|\nabla u|^{2 p(x)-2} \nabla u}{\sqrt{1+|\nabla u|^{2 p(x)}}}\right) \nabla v d x=\int_{\Omega} f(x, u) v d x
$$

for any $v \in W_{0}^{1, p(x)}(\Omega)$. The energy functional $I: W_{0}^{1, p(x)}(\Omega) \rightarrow \mathbb{R}$ corresponding to the problem $(\mathbf{P})$ is

$$
I(u)=\mathcal{M}(L(u))-\int_{\Omega} F(x, u) d x
$$

where $\mathcal{M}(t)=\int_{0}^{t} M(\xi) d \xi$ and $F(x, u)=\int_{0}^{u} f(x, \varphi) d \varphi$.

Thanks to the conditions $\left(\mathrm{M}_{0}\right)$ and $\left(\mathrm{f}_{0}\right)$ (see below), the functional $I$ is well defined and of class $C^{1}$. Since the problem $(\mathbf{P})$ is in the variational setting, the critical points of $I$ are weak solutions of $(\mathbf{P})$. Moreover, the derivative of $I$ is the mapping $I^{\prime}: W_{0}^{1, p(x)}(\Omega) \rightarrow\left(W_{0}^{1, p(x)}(\Omega)\right)^{*}$ given by the formula

$$
\left\langle I^{\prime}(u), v\right\rangle=M(L(u)) \int_{\Omega}\left(|\nabla u|^{p(x)-2} \nabla u+\frac{|\nabla u|^{2 p(x)-2} \nabla u}{\sqrt{1+|\nabla u|^{2 p(x)}}}\right) \nabla v d x-\int_{\Omega} f(x, u) v d x
$$

for any $u, v \in W_{0}^{1, p(x)}(\Omega)$, where

$$
\int_{\Omega}\left(|\nabla u|^{p(x)-2} \nabla u+\frac{|\nabla u|^{2 p(x)-2} \nabla u}{\sqrt{1+|\nabla u|^{2 p(x)}}}\right) \nabla v d x:=\left\langle L^{\prime}(u), v\right\rangle=L^{\prime}(u) v .
$$




\section{Main results}

Theorem 8 Assume the following conditions hold:

$\left(\mathrm{M}_{0}\right) M: \mathbb{R}^{+} \rightarrow \mathbb{R}^{+}$is a continuous function and satisfies the condition

$$
m_{0} t^{\alpha-1} \leq M(t)
$$

for all $t>0$, where $m_{0}$ and $\alpha>1$ are positive real numbers;

$\left(\mathrm{f}_{0}\right) \quad f: \bar{\Omega} \times \mathbb{R} \rightarrow \mathbb{R}$ satisfies the Carathéodory condition and there exist positive constants $C_{2}$ and $C_{3}$ such that

$$
|f(x, t)| \leq C_{2}+C_{3}|t|^{q(x)-1}
$$

for all $x \in \bar{\Omega}$ and $t \in \mathbb{R}$, where $p, q \in C_{+}(\bar{\Omega})$ such that $q^{+}<\alpha p^{-}<p^{*}(x)$. Then $(\mathbf{P})$ has a weak solution.

Proof By the assumptions $\left(\mathrm{M}_{0}\right)$ and $\left(\mathrm{f}_{0}\right)$, we have

$$
\begin{aligned}
I(u) & =\mathcal{M}(L(u))-\int_{\Omega} F(x, u) d x \\
& \geq m_{0} \int_{0}^{L(u)} \xi^{\alpha-1} d \xi-\int_{\Omega} F(x, u) d x \\
& \geq \frac{m_{0}}{\alpha}(L(u))^{\alpha}-\frac{C_{4}}{q^{-}} \int_{\Omega}|u|^{q(x)} d x-C_{4} .
\end{aligned}
$$

Therefore, by Proposition 3 and Proposition 5, it follows

$$
\begin{aligned}
I(u) & \geq \frac{m_{0}}{\alpha\left(p^{+}\right)^{\alpha}}\left(\|u\|^{p^{-}}+\sqrt{1+\|u\|^{2 p^{-}}}\right)^{\alpha}-\frac{C_{4}}{q^{-}}\|u\|^{q^{+}}-C_{4} \\
& \geq \frac{2^{\alpha} m_{0}}{\alpha\left(p^{+}\right)^{\alpha}}\|u\|^{\alpha p^{-}}-\frac{C_{4}}{q^{-}}\|u\|^{q^{+}}-C_{4} \rightarrow+\infty \quad \text { as }\|u\| \rightarrow \infty .
\end{aligned}
$$

By the assumption $q^{+}<\alpha p^{-}, I$ is coercive. Since $I$ is weakly lower semicontinuous, $I$ has a minimum point $u$ in $W_{0}^{1, p(x)}(\Omega)$ and $u$ is a weak solution of $(\mathbf{P})$.

Theorem 9 Assume the following conditions hold:

$\left(\mathrm{M}_{1}\right) M: \mathbb{R}^{+} \rightarrow \mathbb{R}^{+}$is a continuous function and satisfies the condition

$$
m_{1} t^{\alpha-1} \leq M(t) \leq m_{2} t^{\alpha-1}
$$

for all $t>0$, where $m_{1}, m_{2}$ and $\alpha$ real numbers such that $0<m_{1} \leq m_{2}$ and $\alpha>1$;

$\left(\mathrm{M}_{2}\right) M$ satisfies

$$
\mathcal{M}(t) \geq M(t) t
$$

for all $t>0$; 
$\left(\mathrm{f}_{1}\right) \quad f: \bar{\Omega} \times \mathbb{R} \rightarrow \mathbb{R}$ satisfies the Carathéodory condition and there exist positive constants $C_{5}$ and $C_{6}$ such that

$$
|f(x, t)| \leq C_{5}+C_{6}|t|^{\beta(x)-1}
$$

for all $x \in \bar{\Omega}$ and $t \in \mathbb{R}$, where $\beta \in C_{+}(\bar{\Omega})$ such that $\beta(x)<p^{*}(x)$ for all $x \in \bar{\Omega}$ and $\alpha p^{+}<\beta^{-}$

$\left(\mathrm{f}_{2}\right) \quad f(x, t)=o\left(|t|^{\alpha p^{+}-1}\right), t \rightarrow 0$ uniformly for $x \in \bar{\Omega}$;

$\left(\mathrm{f}_{3}\right) \quad$ There exists $t_{*}>0$ such that $F(x, t)>0$ for $x \in \bar{\Omega}$ and all $t \geq t_{*}$;

(AR) Ambrosetti-Rabinowitz's condition holds, i.e., $\exists t_{*}>0, \theta>\frac{m_{2}}{m_{1}} \alpha p^{+}$such that

$$
0 \leq \theta F(x, t) \leq f(x, t) t, \quad|t| \geq t_{*} \text { a.e. } x \in \bar{\Omega} \text {. }
$$

Then (P) has at least one nontrivial weak solution.

To obtain the result of Theorem 9, we need to show that Lemma 10 and Lemma 11 hold.

Lemma 10 Suppose $\left(\mathrm{M}_{1}\right),\left(\mathrm{M}_{2}\right),(\mathrm{AR})$ and $\left(\mathrm{f}_{1}\right)$ hold. Then I satisfies the (PS) condition.

Proof Let us assume that there exists a sequence $\left\{u_{n}\right\}$ in $W_{0}^{1, p(x)}(\Omega)$ such that

$$
I\left(u_{n}\right) \rightarrow c \text { and } I^{\prime}\left(u_{n}\right) \rightarrow 0
$$

Then

$$
\begin{aligned}
c+\left\|u_{n}\right\| & \geq I\left(u_{n}\right)-\frac{1}{\theta} I^{\prime}\left(u_{n}\right) u_{n} \\
& =\mathcal{M}\left(L\left(u_{n}\right)\right)-\frac{1}{\theta} M\left(L\left(u_{n}\right)\right) L^{\prime}\left(u_{n}\right) u_{n}+\int_{\Omega}\left(\frac{1}{\theta} f\left(x, u_{n}\right) u_{n}-F\left(x, u_{n}\right)\right) d x .
\end{aligned}
$$

Since $\sqrt{1+|\nabla u|^{2 p(x)}} \geq|\nabla u|^{p(x)}$, we have $L\left(u_{n}\right) \geq \frac{2}{p^{+}} \int_{\Omega}\left|\nabla u_{n}\right|^{p(x)} d x$. Therefore,

$$
\begin{aligned}
L^{\prime}\left(u_{n}\right) u_{n} & =\int_{\Omega}\left(\left|\nabla u_{n}\right|^{p(x)-2} \nabla u_{n}+\frac{\left|\nabla u_{n}\right|^{2 p(x)-2} \nabla u_{n}}{\sqrt{1+\left|\nabla u_{n}\right|^{2 p(x)}}}\right) \nabla u_{n} d x \\
& \leq 2 \int_{\Omega}\left|\nabla u_{n}\right|^{p(x)} d x \leq p^{+} L\left(u_{n}\right) .
\end{aligned}
$$

By the above inequalities and assumptions $\left(M_{1}\right),\left(M_{2}\right)$ and $(A R)$, we get

$$
\begin{aligned}
c+\left\|u_{n}\right\| & \geq M\left(L\left(u_{n}\right)\right) L\left(u_{n}\right)-\frac{p^{+}}{\theta} M\left(L\left(u_{n}\right)\right) L\left(u_{n}\right)-c \\
& \geq\left(1-\frac{p^{+}}{\theta}\right) M\left(L\left(u_{n}\right)\right) L\left(u_{n}\right)-c \\
& \geq\left(\frac{\theta-p^{+}}{\theta}\right) L\left(u_{n}\right)^{\alpha-1} L\left(u_{n}\right)-c \\
& \geq \frac{2^{\alpha}\left(\theta-p^{+}\right)}{\theta\left(p^{+}\right)^{\alpha}}\left\|u_{n}\right\|^{\alpha p^{-}}-c .
\end{aligned}
$$


This implies that $\left\{\left\|u_{n}\right\|\right\}$ is bounded in $W_{0}^{1, p(x)}(\Omega)$. Passing to a subsequence if necessary, there exists $u \in W_{0}^{1, p(x)}(\Omega)$ such that $u_{n} \rightarrow u$. Therefore, by Proposition 5 , we have

$$
\begin{array}{ll}
u_{n} \rightarrow u & \text { in } W_{0}^{1, p(x)}(\Omega), \\
u_{n} \rightarrow u & \text { in } L^{\beta(x)}(\Omega), \\
u_{n} \rightarrow u & \text { a.e. in } \Omega .
\end{array}
$$

By (3.2), we have $\left\langle I^{\prime}\left(u_{n}\right), u_{n}-u\right\rangle \rightarrow 0$. Thus

$$
\begin{aligned}
& \left\langle I^{\prime}\left(u_{n}\right), u_{n}-u\right\rangle \\
& \quad=M\left(L\left(u_{n}\right)\right) \int_{\Omega}\left(\left|\nabla u_{n}\right|^{p(x)-2} \nabla u_{n}+\frac{\left|\nabla u_{n}\right|^{2 p(x)-2} \nabla u_{n}}{\sqrt{1+\left|\nabla u_{n}\right|^{2 p(x)}}}\right) \nabla\left(u_{n}-\nabla u\right) d x \\
& \quad-\int_{\Omega} f\left(x, u_{n}\right)\left(u_{n}-u\right) \rightarrow 0 .
\end{aligned}
$$

From $\left(f_{1}\right)$ and Proposition 1 , it follows

$$
\begin{aligned}
& \left|\int_{\Omega} f\left(x, u_{n}\right)\left(u_{n}-u\right) d x\right| \\
& \quad \leq\left. C_{6}\left|\int_{\Omega}\right| u_{n}\right|^{\beta(x)-2} u_{n}\left(u_{n}-u\right) d x\left|+C_{5}\right| \int_{\Omega}\left(u_{n}-u\right) d x \mid \\
& \quad \leq\left. C_{6}|| u_{n}\right|^{\beta(x)-1}\left|\frac{\beta(x)}{\beta(x)-1}\right| u_{n}-\left.u\right|_{\beta(x)}+C_{5} \int_{\Omega}\left|u_{n}-u\right| d x .
\end{aligned}
$$

If we consider the relations given in (3.3), we get

$$
\int_{\Omega} f\left(x, u_{n}\right)\left(u_{n}-u\right) d x \rightarrow 0
$$

Hence,

$$
M\left(L\left(u_{n}\right)\right) \int_{\Omega}\left(\left|\nabla u_{n}\right|^{p(x)-2} \nabla u_{n}+\frac{\left|\nabla u_{n}\right|^{2 p(x)-2} \nabla u_{n}}{\sqrt{1+\left|\nabla u_{n}\right|^{2 p(x)}}}\right) \nabla\left(u_{n}-\nabla u\right) d x \rightarrow 0 .
$$

From $\left(M_{1}\right)$, we get

$$
\int_{\Omega}\left(\left|\nabla u_{n}\right|^{p(x)-2} \nabla u_{n}+\frac{\left|\nabla u_{n}\right|^{2 p(x)-2} \nabla u_{n}}{\sqrt{1+\left|\nabla u_{n}\right|^{2 p(x)}}}\right)\left(\nabla u_{n}-\nabla u\right) d x \rightarrow 0
$$

Since the functional (3.4) is of type $\left(S_{+}\right)$(see Proposition 3.1 in [6]), we get $u_{n} \rightarrow u$ in $W_{0}^{1, p(x)}(\Omega)$. We are done.

Lemma 11 Suppose $\left(\mathrm{M}_{1}\right),(\mathrm{AR})$ and $\left(\mathrm{f}_{1}\right)-\left(\mathrm{f}_{3}\right)$ hold. Then the following statements hold:

(i) There exist two positive real numbers $\gamma$ and a such that $I(u) \geq a>0, u \in W_{0}^{1, p(x)}(\Omega)$ with $\|u\|=\gamma$;

(ii) There exists $u \in W_{0}^{1, p(x)}(\Omega)$ such that $\|u\|>\gamma, I(u)<0$. 
Proof (i) Let $\|u\|<1$. Then by $\left(\mathrm{M}_{1}\right)$ and Proposition 3 , we have

$$
I(u) \geq \frac{2^{\alpha} m_{1}}{\alpha\left(p^{+}\right)^{\alpha}}\|u\|^{\alpha p^{+}}-\int_{\Omega} F(x, u) d x .
$$

Since $\alpha p^{+}<\beta^{-}<p^{*}(x)$, by Proposition 5 we have the continuous embeddings $W_{0}^{1, p(x)}(\Omega) \hookrightarrow L^{\alpha p^{+}}(\Omega)$ and $W_{0}^{1, p(x)}(\Omega) \hookrightarrow L^{\beta^{+}}(\Omega) \hookrightarrow L^{\beta^{-}}(\Omega)$, and also there are positive constants $C_{7}, C_{8}$ and $C_{9}$ such that

$$
|u|_{\alpha p^{+}} \leq C_{7}\|u\|, \quad \forall u \in W_{0}^{1, p(x)}(\Omega)
$$

and

$$
|u|_{\beta^{-}} \leq C_{8}\|u\|, \quad|u|_{\beta^{+}} \leq C_{9}\|u\|, \quad \forall u \in W_{0}^{1, p(x)}(\Omega) .
$$

From $\left(\mathrm{f}_{1}\right)$ and $\left(\mathrm{f}_{2}\right)$, we get $F(x, t) \leq \varepsilon|t|^{\alpha p^{+}}+C_{\varepsilon}|t|^{\beta(x)}$ for all $x \in \bar{\Omega}$ and $t \in \mathbb{R}$, where $\varepsilon>0$ is small enough and $C_{\varepsilon}>0$. Therefore, by $\left(\mathrm{M}_{1}\right)$, Proposition 3 and (3.5), (3.6), it follows

$$
\begin{aligned}
I(u) & \geq \frac{2^{\alpha} m_{1}}{\alpha\left(p^{+}\right)^{\alpha}}\|u\|^{\alpha p^{+}}-\varepsilon \int_{\Omega}|u|^{\alpha p^{+}} d x-C_{\varepsilon} \int_{\Omega}|u|^{\beta(x)} d x \\
& \geq \frac{2^{\alpha} m_{1}}{\alpha\left(p^{+}\right)^{\alpha}}\|u\|^{\alpha p^{+}}-\left(\varepsilon|u|_{\alpha p^{+}}^{\alpha p^{+}}+C_{\varepsilon}^{\beta^{+}}|u|_{\beta^{+}}^{\beta^{+}}+C_{\varepsilon}^{\beta^{-}}|u|_{\beta^{-}}^{\beta^{-}}\right) \\
& \geq \frac{2^{\alpha} m_{1}}{\alpha\left(p^{+}\right)^{\alpha}}\|u\|^{\alpha p^{+}}-\varepsilon C_{10}^{\alpha p^{+}}\|u\|^{\alpha p^{+}}-C_{8}^{\beta^{-}}\|u\|^{\beta^{-}}-C_{9}^{\beta^{+}}\|u\|^{\beta^{+}} \\
& \geq\left(\frac{2^{\alpha} m_{1}}{\alpha\left(p^{+}\right)^{\alpha}}-\varepsilon C_{10}^{\alpha p^{+}}\right)\|u\|^{\alpha p^{+}}-\max \left\{C_{8}^{\beta^{-}}, C_{9}^{\beta^{+}}\right\}\|u\|^{\beta^{-}}
\end{aligned}
$$

providing that $\varepsilon C_{10}^{\alpha p^{+}}<\frac{m_{1}}{2 \alpha\left(p^{+}\right)^{\alpha}}$. Since $\|u\|<1$ and $\alpha p^{+}<\beta^{-}$, there exist two positive real numbers $\gamma$ and $a$ such that $I(u) \geq a>0, u \in W_{0}^{1, p(x)}(\Omega)$ with $\|u\|=\gamma \in(0,1)$.

(ii) From (AR) and $\left(f_{3}\right)$, one easily deduces

$$
F(x, t) \geq \frac{F\left(x, t_{*}\right)}{t_{*}^{\theta}} t_{*}^{\theta}
$$

for all $x \in \bar{\Omega}$ and $t \geq t_{*}$. Therefore, for $\delta>1$ and nonnegative $u \in W_{0}^{1, p(x)}(\Omega)$ such that $\left\{x \in \bar{\Omega}: u(x) \geq t_{*}\right\}$, we get

$$
\begin{aligned}
\int_{\Omega} F(x, \delta u) d x & \geq \int_{\left\{\delta u \geq t_{*}\right\}} F(x, \delta u) d x \geq \frac{\delta^{\theta}}{t_{*}^{\theta}} \int_{\left\{\delta u \geq t_{*}\right\}} F\left(x, t_{*}\right) u^{\theta} d x \\
& \geq \frac{\delta^{\theta}}{t_{*}^{\theta}} \int_{\left\{u \geq t_{*}\right\}} F\left(x, t_{*}\right) u^{\theta} d x \geq \delta^{\theta} \int_{\left\{u \geq t_{*}\right\}} F\left(x, t_{*}\right) d x>0
\end{aligned}
$$

(recall that $F \geq 0$ and $F\left(\cdot, t_{*}\right)>0$ almost everywhere). On the other hand, when $t>t_{*}$, from $\left(\mathrm{M}_{1}\right)$ we obtain that

$$
\mathcal{M}(t) \leq \frac{m_{2}}{\alpha} t^{\alpha} \leq \frac{m_{2}}{\alpha} t^{\frac{m_{2}}{m_{1}} \alpha}
$$


Since $t>1$, it is obvious $L(t \omega) \leq t^{p^{+}} L(\omega)$. Hence, for $\omega \in W_{0}^{1, p(x)}(\Omega) \backslash\{0\}$, we have

$$
\begin{aligned}
I(t \omega) & =\mathcal{M}(L(t \omega))-\int_{\Omega} F(x, t \omega) d x \\
& \leq \frac{m_{2}}{\alpha}(L(t \omega))^{\frac{m_{2}}{m_{1}} \alpha}-\int_{\Omega} F(x, t \omega) d x \\
& \leq \frac{m_{2}}{\alpha} t^{\frac{m_{2}}{m_{1}} \alpha p^{+}}(L(\omega))^{\frac{m_{2}}{m_{1}} \alpha}-t^{\theta} \int_{\left\{\omega \geq t_{*}\right\}} F(x, \omega) d x .
\end{aligned}
$$

From the assumption on $\theta$ (see (AR)), we conclude $I(t \omega) \rightarrow-\infty$ as $t \rightarrow+\infty$.

Proof of Theorem 9 From Lemma 10, Lemma 11 and the fact that $I(0)=0, I$ satisfies the mountain pass theorem (see $[25,26])$. Therefore, $I$ has at least one nontrivial weak solution. The proof of Theorem 9 is completed.

In the sequel, using Krasnoselskii's genus theory (see [25, 27]), we show the existence of infinitely many solutions of the problem $(\mathbf{P})$. So, we recall some basic notations of Krasnoselskii's genus.

Let $X$ be a real Banach space and set

$$
\Re=\{E \subset X \backslash\{0\}: E \text { is compact and } E=-E\} .
$$

Definition 12 Let $E \in \mathfrak{R}$ and $X=\mathbb{R}^{k}$. The genus $\gamma(E)$ of $E$ is defined by

$$
\gamma(E)=\min \left\{k \geq 1 \text {; there exists an odd continuous mapping } \phi: E \rightarrow \mathbb{R}^{k} \backslash\{0\}\right\}
$$

If such a mapping does not exist for any $k>0$, we set $\gamma(E)=\infty$. Note also that if $E$ is a subset which consists of finitely many pairs of points, then $\gamma(E)=1$. Moreover, from the definition, $\gamma(\varnothing)=0$. A typical example of a set of genus $k$ is a set which is homeomorphic to a $(k-1)$ dimensional sphere via an odd map.

Now, we will give some results of Krasnoselskii's genus which are necessary throughout the present paper.

Theorem 13 Let $X=\mathbb{R}^{N}$ and $\partial \Omega$ be the boundary of an open, symmetric and bounded subset $\Omega \subset \mathbb{R}^{N}$ with $0 \in \Omega$. Then $\gamma(\partial \Omega)=N$.

Corollary $14 \gamma\left(S^{N-1}\right)=N$.

Remark 15 If $X$ is of an infinite dimension and separable and $S$ is the unit sphere in $X$, then $\gamma(S)=\infty$.

Theorem 16 Suppose that $M$ and f satisfy the following conditions:

$\left(\mathrm{M}_{3}\right) M: \mathbb{R}^{+} \rightarrow \mathbb{R}^{+}$is a continuous function and satisfies the condition

$$
m_{3} t^{\delta-1} \leq M(t) \leq m_{4} t^{\alpha-1}
$$

for all $t>0$, where $m_{3}, m_{4}, \delta$ and $\alpha$ are real numbers such that $0<m_{3} \leq m_{4}$ and $1<\delta \leq \alpha$ 
$\left(\mathrm{f}_{4}\right) \quad f: \bar{\Omega} \times \mathbb{R} \rightarrow \mathbb{R}$ is a continuous function and there exist positive constants $C_{11}, C_{12}, C_{13}$ and $C_{14}$ such that

$$
C_{11}+C_{12}|t|^{s(x)-1} \leq f(x, t) \leq C_{13}+C_{14}|t|^{q(x)-1}
$$

for all $x \in \bar{\Omega}$ and $t \geq 0$, where $s, q \in C(\bar{\Omega})$ such that $1<s(x)<q(x)<p^{*}(x)$ for all $x \in \bar{\Omega}$;

$\left(\mathrm{f}_{5}\right) \quad f$ is an odd function according to $t$, that is,

$$
f(x, t)=-f(x,-t)
$$

for all $x \in \bar{\Omega}$ and $t \in \mathbb{R}$.

If $p(x)<q(x)<p^{*}(x)$ for all $x \in \bar{\Omega}$ and $q^{+}<\delta p^{-}$, then the problem $(\mathbf{P})$ has infinitely many solutions.

The following result obtained by Clarke in [28] is the main idea which we use in the proof of Theorem 16 .

Theorem 17 Let $J \in C^{1}(X, \mathbb{R})$ be a functional satisfying the (PS) condition. Furthermore, let us suppose that:

(i) $J$ is bounded from below and even;

(ii) There is a compact set $K \in \mathfrak{R}$ such that $\gamma(K)=k$ and $\sup _{x \in K} J(x)<J(0)$.

Then $J$ possesses at least $k$ pairs of distinct critical points and their corresponding critical values are less than $J(0)$.

Lemma 18 Suppose $\left(\mathrm{M}_{3}\right),\left(\mathrm{f}_{4}\right)$ and the inequality $q^{+}<\delta p^{-}$hold.

(i) I is bounded from below;

(ii) I satisfies the (PS) condition.

Proof (i) By the assumptions $\left(\mathrm{M}_{3}\right)$ and $\left(\mathrm{f}_{4}\right)$, we have

$$
\begin{aligned}
I(u) & =\mathcal{M}(L(u))-\int_{\Omega} F(x, u) d x \\
& \geq m_{3} \int_{0}^{L(u)} \xi^{\delta-1} d \xi-\frac{C_{14}}{q^{-}} \int_{\Omega}|u|^{q(x)} d x-C_{13} \\
& \geq \frac{2^{\delta} m_{3}}{\delta\left(p^{+}\right)^{\delta}}\|u\|^{\delta p^{+}}-\frac{C_{14}}{q^{-}} \int_{\Omega}|u|^{q(x)} d x-C_{13} .
\end{aligned}
$$

By Proposition 3 and Proposition 5, we get

$$
\begin{aligned}
I(u) & \geq \frac{2^{\delta} m_{3}}{\delta\left(p^{+}\right)^{\delta}}\|u\|^{\delta p^{-}}-\frac{C_{14}}{q^{-}} \max \left\{|u|_{q(x)}^{q^{-}},|u|_{q(x)}^{q^{+}}\right\}-C_{13} \\
& \geq \frac{2^{\delta} m_{3}}{\delta\left(p^{+}\right)^{\delta}}\|u\|^{\delta p^{-}}-\frac{C_{14}}{q^{-}} \max \left\{C^{q^{-}}\|u\|^{q^{-}}, C^{q^{+}}\|u\|^{q^{+}}\right\}-C_{13} \\
& \geq \frac{2^{\delta} m_{3}}{\delta\left(p^{+}\right)^{\delta}}\|u\|^{\delta p^{-}}-\frac{C_{14}}{q^{-}} C^{q^{+}}\|u\|^{q^{+}}-C_{13}
\end{aligned}
$$

for $\|u\|$ large enough. Hence, $I$ is bounded from below. 
(ii) Let us assume that there exists a sequence $\left\{u_{n}\right\}$ in $W_{0}^{1, p(x)}(\Omega)$ such that

$$
I\left(u_{n}\right) \rightarrow c \quad \text { and } \quad I^{\prime}\left(u_{n}\right) \rightarrow 0 .
$$

From (3.8) we have $\left|I\left(u_{n}\right)\right| \leq C_{16}$. This fact combined with (3.7) implies that

$$
C_{16} \geq I\left(u_{n}\right) \geq \frac{2^{\delta} m_{3}}{\delta\left(p^{+}\right)^{\delta}}\|u\|^{\delta p^{-}}-C_{15}\|u\|^{q^{+}} \geq C_{17}
$$

where $\left\|u_{n}\right\|>1$. Since $q^{+}<\delta p^{-}$, we obtain that $\left\{\left\|u_{n}\right\|\right\}$ is bounded in $W_{0}^{1, p(x)}(\Omega)$.

Hence, we may extract a subsequence $\left\{u_{n}\right\} \subset W_{0}^{1, p(x)}(\Omega)$ and $u \in W_{0}^{1, p(x)}(\Omega)$ such that $u_{n} \rightarrow u$ in $W_{0}^{1, p(x)}(\Omega)$. In the rest of the proof, if we consider similar relations given in (3.3) and growth conditions assumed on $f$ and apply the same processes which we used in the proof of Lemma 10, we can see that $I$ satisfies the (PS) condition.

Proof of Theorem 16 Set (see $[7,25])$

$$
\begin{aligned}
& \mathfrak{R}_{k}=\{E \subset \mathfrak{R}: \gamma(E) \geq k\}, \\
& c_{k}=\inf _{E \in \mathfrak{R}_{k}} \sup _{u \in E} I(u), \quad k=1,2, \ldots,
\end{aligned}
$$

then we have

$$
-\infty<c_{1} \leq c_{2} \leq \cdots \leq c_{k} \leq c_{k+1} \leq \cdots
$$

Now, we will show that $c_{k}<0$ for every $k \in \mathbb{N}$. Since $W_{0}^{1, p(x)}(\Omega)$ is a reflexive and separable Banach space, for any $k \in \mathbb{N}$, we can choose a $k$-dimensional linear subspace $X_{k}$ of $W_{0}^{1, p(x)}(\Omega)$ such that $X_{k} \subset C_{0}^{\infty}(\Omega)$. As the norms on $X_{k}$ are equivalent, there exists $r_{k} \in(0,1)$ such that $u \in X_{k}$ with $\|u\| \leq r_{k}$ implies $|u|_{L^{\infty}} \leq \delta$.

Set $S_{r_{k}}^{(k)}=\left\{u \in X_{k}:\|u\|=r_{k}\right\}$. By the compactness of $S_{r_{k}}^{(k)}$ and the condition $\left(\mathrm{f}_{4}\right)$, there exists a constant $\eta_{k}>0$ such that

$$
\begin{aligned}
& F(x, u) \geq C_{11}+C_{12}|t|^{s(x)}, \\
& \int_{\Omega} F(x, u) d x \geq \frac{C_{12}}{s^{+}} \int_{\Omega}|u|^{s(x)} d x+C_{11} \int_{\Omega} d x \geq \eta_{k}+C_{18}
\end{aligned}
$$

for all $u \in S_{r_{k}}^{(k)}$. If we consider $\left(\mathrm{M}_{3}\right)$ and $\left(\mathrm{f}_{4}\right)$, for $u \in S_{r_{k}}^{(k)}$ and $t \in(0,1)$, we have

$$
\begin{aligned}
I(t u) & =\mathcal{M}(L(t u))-\int_{\Omega} F(x, t u) d x \leq \frac{m_{4}}{\alpha}(L(t u))^{\alpha}-t^{s^{+}} \eta_{k}-C_{18} \\
& \leq \frac{m_{4}}{\alpha}\left(\int_{\Omega} \frac{|\nabla t u|^{p(x)}+\sqrt{1+|\nabla t u|^{2 p(x)}}}{p(x)} d x\right)^{\alpha}-t^{s^{+}} \eta_{k}-C_{18} \\
& \leq \frac{m_{4}}{\alpha\left(p^{-}\right)^{\alpha}}\left(t^{p^{-}}\|u\|^{p^{-}}+1+t^{p^{-}}\|u\|^{p^{-}}\right)^{\alpha}-t^{s^{+}} \eta_{k}-C_{18} \\
& \leq \frac{m_{4}}{\alpha\left(p^{-}\right)^{\alpha}} 2^{\alpha-1}\left(\left(2 t^{p^{-}}\|u\|^{p^{-}}\right)^{\alpha}+1\right)-t^{s^{+}} \eta_{k}-C_{18} \\
& \leq \frac{m_{4}}{\alpha\left(p^{-}\right)^{\alpha}} 2^{2 \alpha-1} t^{\alpha p^{-}} r_{k}^{\alpha p^{-}}-t^{s^{+}} \eta_{k}+\frac{m_{4}}{\alpha\left(p^{-}\right)^{\alpha}} 2^{\alpha-1}-C_{18},
\end{aligned}
$$


providing that $C_{18} \geq \frac{m_{4}}{\alpha\left(p^{-}\right)^{\alpha}} 2^{\alpha-1}$. Since $s^{+}<q^{-} \leq q^{+}<\delta p^{-} \leq \alpha p^{-}$, we can find $t_{k} \in(0,1)$ and $\varepsilon_{k}>0$ such that

$$
I\left(t_{k} u\right) \leq-\varepsilon_{k}<0 \quad \text { for all } u \in S_{r_{k}}^{(k)}
$$

i.e.,

$$
I(u) \leq-\varepsilon_{k}<0 \quad \text { for all } u \in S_{t_{k} r_{k}}^{(k)}
$$

It is clear that $\gamma\left(S_{t_{k} r_{k}}^{(k)}\right)=k$, so $c_{k} \leq-\varepsilon_{k}<0$. Finally, by Lemma 18 above, we can apply Theorem 17 to obtain that the functional $I$ admits at least $k$ pairs of distinct critical points, and since $k$ is arbitrary, we obtain infinitely many critical points of $I$. The proof is completed.

Theorem 19 Suppose $\left(\mathrm{M}_{3}\right),\left(\mathrm{f}_{4}\right)$ and $\left(\mathrm{f}_{5}\right)$ hold. If $q(x)<p(x)<p^{*}(x)$ for all $x \in \bar{\Omega}$, then the problem $(\mathbf{P})$ has a sequence of solutions $\left\{ \pm u_{k}: k=1,2, \ldots\right\}$ such that $I\left( \pm u_{k}\right)<0$.

Proof In the beginning, we will show that $I$ is coercive. If we follow the same processes applied in the proof of Theorem 8 and consider the fact $q^{+}<p^{-}$, it is easy to get the coerciveness of $I$. Since $I$ is weak lower semi-continuous, $I$ attains its minimum on $W_{0}^{1, p(x)}(\Omega)$, i.e., (P) has a solution. By help of coerciveness, we know that $I$ satisfies the (PS) condition on $W_{0}^{1, p(x)}(\Omega)$. Moreover, from the condition $\left(f_{5}\right), I$ is even.

In the rest of the proof, since we develop the same arguments which we used in the proof of Theorem 16, we omit the details. Therefore, if we follow similar steps to those in (3.9) and (3.10) and consider the inequalities $s^{+}<q^{-} \leq q^{+}<p^{-}<\alpha p^{-}$, we can find $t_{k} \in(0,1)$ and $\varepsilon_{k}>0$ such that

$$
I(u) \leq-\varepsilon_{k}<0 \quad \text { for all } u \in S_{t_{k} r_{k}}^{(k)}
$$

Obviously, $\gamma\left(S_{t_{k} r_{k}}^{(k)}\right)=k$, so $c_{k} \leq-\varepsilon_{k}<0$. By Krasnoselskii's genus, each $c_{k}$ is a critical value of $I$, hence there is a sequence of solutions $\left\{ \pm u_{k}: k=1,2, \ldots\right\}$ such that $I\left( \pm u_{k}\right)<0$.

\section{Competing interests}

The author declares that he has no competing interests.

\section{Acknowledgements}

The author would like to thank the referee for some valuable comments and helpful suggestions.

Received: 28 December 2012 Accepted: 28 February 2013 Published: 18 March 2013

\section{References}

1. Ni, WM, Serrin, J: Non-existence theorems for quasilinear partial differential equations. Rend. Circ. Mat. Palermo Suppl. 8, 171-185 (1985)

2. Ni, WM, Serrin, J: Existence and non-existence theorems for ground states for quasilinear partial differential equations. Atti Conv. Lincei 77, 231-257 (1985)

3. Concus, P, Finn, P: A singular solution of the capillary equation I, II. Invent. Math. 29(143-148), 149-159 (1975)

4. Finn, R: On the behavior of a capillary surface near a singular point. J. Anal. Math. 30, 156-163 (1976)

5. Johnson, WE, Perko, L: Interior and exterior boundary value problems from the theory of the capillary tube. Arch. Ration. Mech. Anal. 29, 129-143 (1968)

6. Rodrigues, MM: Multiplicity of solutions on a nonlinear eigenvalue problem for $p(x)$-Laplacian-like operators. Mediterr. J. Math. 9(1), 211-223 (2012)

7. Avci, M, Cekic, B, Mashiyev, RA: Existence and multiplicity of the solutions of the $p(x)$-Kirchhoff type equation via genus theory. Math. Methods Appl. Sci. 34(14), 1751-1759 (2011) 
8. Dai, G, Hao, R: Existence of solutions for a p(x)-Kirchhoff-type equation. J. Math. Anal. Appl. 359, $275-284$ (2009)

9. Fan, XL: On nonlocal $p(x)$-Laplacian Dirichlet problems. Nonlinear Anal. 72, 3314-3323 (2010)

10. Kirchhoff, G: Mechanik. Teubner, Leipzig (1883)

11. Antontsev, SN, Shmarev, SI: A model porous medium equation with variable exponent of nonlinearity: existence, uniqueness and localization properties of solutions. Nonlinear Anal. 60, 515-545 (2005)

12. Antontsev, SN, Rodrigues, JF: On stationary thermo-rheological viscous flows. Ann. Univ. Ferrara, Sez. 7: Sci. Mat. 52, 19-36 (2006)

13. Halsey, TC: Electrorheological fluids. Science 258, 761-766 (1992)

14. Růžička, M: Electrorheological Fluids: Modeling and Mathematical Theory. Lecture Notes in Mathematics. Springer, Berlin (2000)

15. Zhikov, W: Averaging of functionals of the calculus of variations and elasticity theory. Math. USSR, Izv. 9, 33-66 (1987)

16. Avci, M: Existence and multiplicity of solutions for Dirichlet problems involving the $p(x)$-Laplace operator. Electron. J. Differ. Equ. 2013(14), 1-9 (2013)

17. Cekic, B, Mashiyev, RA: Existence and localization results for $p(x)$-Laplacian via topological methods. Fixed Point Theory Appl. 2010, Article ID 120646 (2010)

18. Fan, XL, Zhang, QH: Existence of solutions for $p(x)$-Laplacian Dirichlet problems. Nonlinear Anal. 52, 1843-1852 (2003)

19. Fan, XL: Eigenvalues of the $p(x)$-Laplacian Neumann problems. Nonlinear Anal. 67(10), 2982-2992 (2007)

20. Mihăilescu, M, Rădulescu, V: On a nonhomogeneous quasilinear eigenvalue problem in Sobolev spaces with variable exponent. Proc. Am. Math. Soc. 135(9), 2929-2937 (2007)

21. Diening, L, Harjulehto, P, Hästö, P, Růžička, M: Lebesgue and Sobolev Spaces with Variable Exponents. Springer, Berlin (2011)

22. Fan, XL, Shen, JS, Zhao, D: Sobolev embedding theorems for spaces $W^{k, p(x)}(\Omega)$. J. Math. Anal. Appl. 262, 749-760 (2001)

23. Fan, $X L$, Zhao, D: On the spaces $L^{p(x)}(\Omega)$ and $W^{m, p(x)}(\Omega)$. J. Math. Anal. Appl. 263, 424-446 (2001)

24. Kovăčik, O, Răkosnik, J: On spaces $L^{p(x)}$ and $W^{k, p(x)}$. Czechoslov. Math. J. 41(116), 592-618 (1991)

25. Chang, KC: Critical Point Theory and Applications. Shanghai Sci. Technol., Shanghai (1986)

26. Willem, M: Minimax Theorems. Birkhäuser, Basel (1996)

27. Krasnoselskii, MA: Topological Methods in the Theory of Nonlinear Integral Equations. MacMillan \& Co., New York (1964)

28. Clarke, DC: A variant of the Lusternik-Schnirelman theory. Indiana Univ. Math. J. 22, 65-74 (1972)

doi:10.1186/1687-2770-2013-55

Cite this article as: Avci: Ni-Serrin type equations arising from capillarity phenomena with non-standard growth. Boundary Value Problems 2013 2013:55.

\section{Submit your manuscript to a SpringerOpen ${ }^{\ominus}$ journal and benefit from:}

- Convenient online submission

Rigorous peer review

- Immediate publication on acceptance

- Open access: articles freely available online

- High visibility within the field

- Retaining the copyright to your article 